\title{
Invasive Therapies for Acute Coronary Syndromes in the COVID-19 Era
}

\author{
Sanket Borgaonkar ${ }^{1} \cdot J_{\text {Joshua Hahn }}{ }^{1} \cdot$ Marilyne Daher $^{1} \cdot$ Waleed Kayani $^{1} \cdot$ Hani Jneid $^{1,2}$ (D) \\ Accepted: 9 March 2021 / Published online: 7 May 2021 \\ (C) This is a U.S. government work and not under copyright protection in the U.S.; foreign copyright protection may apply 2021
}

\begin{abstract}
Purpose of Review To identify and address the challenges associated with the care of ACS patients during the coronavirus 2019 pandemic.

Recent Findings The COVID-19 pandemic has had a considerable global impact with over 2.0 million deaths worldwide so far. There has been considerable evidence suggesting that COVID-19 increases the risk of acute coronary syndromes (ACS).

Summary We propose characterizing ACS patients into 3 distinct categories to better assist in appropriate triage and management: critically ill patients, non-critically ill ST elevation myocardial infarction (STEMI) patients, and non-critically ill non-ST elevation myocardial infarction (NSTEMI)/unstable angina (UA) patients. We thoroughly review treatments strategies, management considerations, and current consensus statements for the care of COVID-19 patients with ACS. As we continue to gain more experience with management of COVID-19 in ACS patients and as health-care workers and patients continue to get vaccinated, we must continue to adapt our strategies to treat this high-risk group of patients.
\end{abstract}

Keywords COVID-19 - Acute coronary syndrome $\cdot$ Cardiac catheterization $\cdot$ Health personnel $\cdot$ Personal protective equipment . ST elevation myocardial infarction

\section{Introduction}

The coronavirus 2019 disease (COVID-19) pandemic caused by the severe acute respiratory syndrome coronavirus 2

This article is part of the Topical Collection on Management of Acute Coronary Syndromes

Hani Jneid

Jneid@bcm.edu

Sanket Borgaonkar

sanket.borgaonkar@bcm.edu

Joshua Hahn

joshua.hahn@bcm.edu

Marilyne Daher

marilyne.daher@bcm.edu

Waleed Kayani

kayani@bcm.edu

1 Division of Cardiology, Baylor College of Medicine, Houston, TX, USA

2 Division of Cardiology, Baylor College of Medicine and the Michael E. DeBakey VA Medical Center Houston, Houston, TX 77030, USA
(SARS-CoV2) has impacted over 180 countries with more than 92 million cases and as of early January 2021 over 1.9 million deaths [1]. Although primarily causing respiratory distress, our continued understanding of the disease has underscored the significant impact of COVID-19 on the cardiovascular system. Indeed, there is growing evidence that COVID-19 increases the risk of acute coronary syndrome (ACS). Data from a nationwide Danish registry suggested that COVID-19 was associated with a fivefold increase in ACS events in the 14-day period following COVID-19 infection [2]. As cases continue to rise, cardiovascular care presents a challenge in COVID-19 positive patients.

The SARS-CoV2 is a novel single-stranded enveloped RNA virus similar to previous zoonotic coronaviruses such as 2002 SARS-CoV (SARS) and the 2012 Middle East respiratory syndrome coronavirus (MERS-CoV) [3]. The SARS$\mathrm{CoV} 2$ infection is caused by the binding of the viral envelope spike protein to the human angiotensin-converting enzyme 2 (ACE2) receptor. While ACE2 receptors are seen in abundance in the alveolar tissue, thus resulting in respiratory symptoms, they are also highly expressed in myocardial tissue and likely play a significant role in COVID-19-related cardiovascular disease. Similar to SARS and MERS, there is an 
increased risk of severe COVID-19 infection in individuals with cardiovascular comorbidities $[4,5]$. In a meta-analysis of 8 studies from Wuhan, China, comprised of 46,248 patients, the most prevalent comorbidity was hypertension and diabetes [6]. When further examining patients with non-severe versus severe COVID-19, cardiovascular disease was significantly more prevalent in patients with severe COVID-19 (OR 3.42, 95\% CI: 1.88-6.22) [6]. Additionally, many studies have shown that there is a significantly higher mortality in patients with COVID-19 and concomitant cardiovascular disease [7,8], with one study of 416 patients from Wuhan, China, reporting as high as $51 \%$ mortality in patients with cardiac injury [9].

\section{Pathophysiology of Covid-19-Related Myocardial Injury and ACS}

The interaction between SARS-CoV2 and the cardiovascular system is bidirectional. While those with pre-existing cardiovascular disease affected by COVID-19 have worse outcomes, the virus itself can induce cardiac injury, and a myriad of other cardiovascular complications, such as arrhythmias, cardiomyopathy and heart failure, and acute coronary syndrome (ACS) [10].

An early case series from China found that $17 \%$ of hospitalized patients and 44\% of ICU patients infected with COVID-19 had an arrhythmia at some point during their hospitalization [11]. A single-center US study of 700 hospitalized patients found that 53 of the 700 patients had an arrhythmic event, of which 25 were atrial fibrillation, 10 were clinically significant brady-arrhythmias, and 9 were cardiac arrests; none of the patients had ventricular tachycardia or ventricular fibrillation [12]. Ventricular arrhythmias were less commonly described in COVID-19 patients. In a Chinese single-center case series of 187 COVID-19 hospitalized patients, 11 (5.9\%) were noted to have a ventricular arrhythmia. While there is sufficient documentation of arrhythmias in COVID-19 patients, it is difficult to attribute any of them directly to SARS-CoV2, given that systemic inflammation, sepsis, fever, and myocardial injury are also known triggers for these arrhythmias.

Unlike arrhythmias, myocardial injury is well described in COVID-19 patients. Up to one-third of COVID-19 patients have myocardial injury, defined as an elevation in cardiac biomarkers of myonecrosis [13]. The presence of myocardial injury in these patients portends a worse prognosis, with one study showing an almost twofold increase in mortality in those with pre-existing cardiovascular comorbidities and myocardial injury compared to those without [8]. A German autopsy study of 39 COVID-19 patients found that $41 \%$ had detectable viral load in their myocardium [14]. Myocarditis has also been described in COVID-19 patients, although mostly in the form of case reports and case series [15-17].
The exact incidence of ACS in patients with COVID-19 is not yet fully known. Compared to the pre-COVID-19 era, statistics from Italy and the USA showed up to a $48 \%$ decline in the rates of hospitalization of patients with ACS $[18,19]$. Despite this, the number of out-of-hospital cardiac arrests increased during the same time period in Italy [20] and in New York City [21]. Other studies showed that outcomes of patients with out-of-hospital cardiac arrests during the COVID19 outbreak were worse than those in pre-COVID-19 times [22]. Using the Universal definition of MI, three types of myocardial infarctions (MI) have been postulated to occur in COVID-19 patients: types 1, 2, and 3. Type $1 \mathrm{MI}$, defined as myocardial infarction due to plaque rupture or erosion, in the setting of COVID-19 is postulated to occur by several mechanisms. At the molecular level, the binding of viral pathogenassociated molecular patterns (PAMPs) to Toll-like receptors (TLRs) leads to a downstream signaling cascade that ultimately causes destabilization of preexisting atherosclerotic plaques and consequent plaque disruption [23]. Systemic infection and inflammation can contribute to ACS by causing endothelial dysfunction and an imbalance in the neurohormonal milieu in favor of thrombosis, vasospasm, and an increase in coagulation factors such as fibrinogen [24]. Type $2 \mathrm{MI}$, defined as a myocardial oxygen supply-demand mismatch [25], has also been noted in COVID-19 patients. Mechanisms for this type of MI include endothelial dysfunction, ACE2 signaling disruption leading to increased angiotensin II levels and subsequent arteriolar vasoconstriction, and hypoxemia secondary to acute respiratory distress syndrome (ARDS) [13]. Type 3 MI, in which sudden death occurs with suspicion for MI but without available cardiac biomarkers [25], is also postulated to occur in COVID-19 patients who had sudden death and known coronary artery disease [13].

While it has been shown that patients with acute coronary syndrome (ACS) and COVID-19 are likely to have a poor prognosis, robust, large-scale data to guide our management is lacking [26]. The management of patients with COVID-19 and ACS, including ST-elevation myocardial infarction (STEMI) and non-ST elevation-ACS (NSTE-ACS) (which includes non-ST elevation myocardial infarction (NSTEMI) and unstable angina (UA)), presents unique challenges. As the number of COVID-19 patients continue to rise, it is important to address a strategy to adequately treat ACS patients while maintaining a systematic approach in assessing COVID-19 patients and limiting patient and health-care worker (HCW) exposure.

\section{Current Consensus Statements}

Major cardiovascular societies and experts across the globe have developed treatment recommendations related to COVID-19 and ACS (Table 1). A common consensus among experts is that in PCI-capable centers, primary PCI remains 
Table 1 Recommendations for acute myocardial infarction management in the COVID-19 pandemic

\author{
Cardiovascular society \\ ACC, SCAI, and ACEP \\ Key recommendations \\ -Initial evaluation in ED \\ -Full HCW PPE \\ - Primary PCI is the preferred strategy in patients with definite STEMI \\ -Primary fibrinolytic therapy can be used if delay in PCI is anticipated \\ -A negative pressure room in the CCL is preferred \\ AHA \\ -Initial evaluation in ED \\ -Primary PCI preferred for clear STEMI \\ -Fibrinolytic therapy can be used if PCI cannot be performed \\ within 120 minutes in STEMI \\ -PPE protocol in CCL should presume all patients COVID positive \\ ESC \\ -Rapid triaging in ED initially \\ -Primary PCI remains therapy of choice in STEMI within 120 min as \\ a goal, however an up to 60-min delay may be permissible during the pandemic \\ -All patients should be considered COVID positive \\ -Fibrinolytics should be administered as soon as this strategy is \\ decided upon in the event that PCI is not feasible in a timely manner
}

ACC American College of Cardiology, SCAI Society for Cardiovascular Angiography and Interventions, ACEP American College of Emergency Physicians, ED emergency department, $H C W$ health-care worker, $P P E$ personal protective equipment, $P C I$ percutaneous coronary intervention, STEMI ST-segment elevation myocardial infarction, $C C L$ cardiac catheterization laboratory, AHA American Heart Association, ESC European Society of Cardiology

the ultimate preferred standard of care for acute STEMI in confirmed or suspected COVID-19 cases when clinical symptoms correlate with classic ECG findings [27-30]. The American College of Cardiology (ACC) and Society for Cardiovascular Angiography and Interventions (SCAI) suggest that in COVID-19 positive patients with STEMI that demonstrate atypical features in either their clinical presentation or ECG, it is reasonable to pursue further investigations (e.g., echocardiogram, serial troponins, and ECGs) prior to deciding on whether to proceed with urgent coronary angiography. Similarly, the European Society of Cardiology (ESC) consensus document suggests that primary PCI may be delayed up to $60 \mathrm{~min}(<120$-min goal) in a COVID-19 positive patient presenting with STEMI before considering alternative strategies (i.e., fibrinolysis). All major societies emphasize a robust COVID-19 triaging system that allows for appropriate resource allocation and choice treatment strategy. Herein, we discuss important management considerations in COVID-19 positive patients presenting with ACS.

\section{Initial Presentation and Evaluation}

The approach to the diagnosis of ACS begins with appropriate triage, and thus, we propose characterizing ACS patients into 3 distinct categories: critically ill STEMI or NSTEMI/ UA, non-critically ill STEMI, and non-critically ill NSTEMI/ UA. All patients, including emergency department, hospitalized, and transferred patients, should undergo a universal screening protocol for COVID-19. Ideally, every patient presenting with ACS should undergo testing for COVID-19; however, this may not always be achievable depending on hospital resources. Notwithstanding, all patients with concerning symptoms should be tested for COVID-19 per the hospital's testing capabilities. Thus, obtaining a thorough history and physical examination to adequately triage patients that may be infected with COVID-19 becomes the critical first step in evaluation. Furthermore, any initial assessment must include a thorough hemodynamic evaluation and electrocardiogram (ECG) review. The extent of myocardial injury can be assessed with serum troponin I levels; however, any plans for emergent reperfusion therapy should not be delayed awaiting troponin levels. Elevated troponin levels have been observed in COVID-19 positive patients, and it is important to differentiate true ACS from other mimickers of myocardial injury [31, 32].

\section{Critically III STEMI or NSTEMI/UA}

Critically ill patients with ACS can be defined as those presenting with cardiogenic shock, profound hemodynamic instability, refractory heart failure, mechanical complications, malignant ventricular arrhythmias, and/or cardiac arrest. The approach to the treatment of critically ill patients with ACS should remain the same as per current practice guidelines [33, 34 ], and includes emergent coronary angiography and 
reperfusion/revascularization (Fig. 1). An emergent invasive approach should be pursued in those presenting with critically ill STEMI or NSTEMI/UA without delay regardless of the patient's COVID-19 status. Concurrently, proper precautions (e.g., advanced personal protective equipment, designated COVID-19 cath lab) must be taken with the assumption that patients are COVID-19 positive.

There are a number of practical and medical considerations when approaching a patient who is presenting with critically ill STEMI or NSTEMI/UA. Timely PCI remains the standard of care for these patients, regardless of COVID-19 status. Some experts have suggested that in the current COVID-19 pandemic, a delay of up to $60 \mathrm{~min}$ is acceptable [29], although not ideal (diagnosis to reperfusion goal of $<120 \mathrm{~min}$ ). Any delay beyond this timeframe may warrant immediate fibrinolytic therapy if there are no contraindications, possibly as part of a pharmaco-invasive approach when feasible (see section on fibrinolytic therapy below).

In general, data are limited regarding the common presenting characteristics of patients with ACS who also have COVID-19. Stefanini et al. [35] investigated 28 patients with STEMI and COVID-19 in Lombardy, Italy, hospitals capable of primary PCI. In their series, all patients underwent coronary angiography; however, 11 patients (39\%) had no evidence of obstructive $\mathrm{CAD}$, and their clinical presentations were attributable to STEMI mimickers, including Type 2 MI, myocarditis, COVID-19-related endothelial dysfunction, and cytokine storm. Similarly, Bangalore et al. [36] observed a significant number of patients who presented with STEMI and COVID-19 did not have obstructive CAD. These observations would

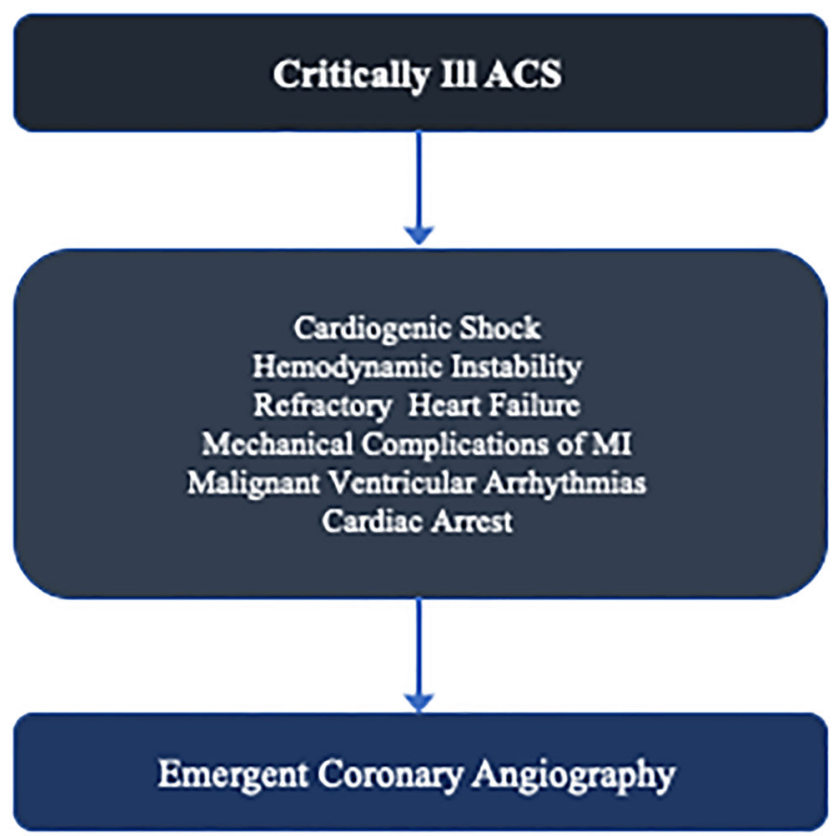

Fig. 1 Management algorithm for critically Ill ACS patients support the pursuit of further investigations including echocardiogram, serial ECGs, and noninvasive coronary CT angiography in patients where the clinical presentation of STEMI is equivocal.

In another retrospective multicenter analysis of 78 patients with STEMI and COVID-19, Hamadeh et al. [37••] found that the majority $(76 \%)$ of these patients received fibrinolytic therapy. Patients' median age was 65 years, and the majority had at least 4 comorbidities with all of them having at least 1 comorbidity. In those that received primary PCI, hypotension requiring vasopressors was noted in over $30 \%$ of patients. Additionally, both cardiopulmonary resuscitation and mechanical ventilation were needed in over $40 \%$ of patients who received primary PCI. These events were observed less frequently in those who received fibrinolytic therapy, which may be due to treatment selection bias with preferential allocation of non-critically ill patients to a fibrinolytic therapy strategy. Most notably, the authors observed a markedly high rate of stent thrombosis (21\%) in those treated with PCI. This finding is consistent with the observed increased rate of thrombotic events in patients with severe COVID-19 [38]. However, it is important to note that these retrospective observational data are hardly conclusive, and the actual rates of stent thrombosis need to be confirmed in larger datasets from multiple PCI capable centers [39]. To date, there are no data from randomized controlled trials to support the routine administration of therapeutic anticoagulation to patients with COVID-19 infection; however, observational data suggesting improved clinical outcomes with empiric anticoagulation exist in patients with severe COVID-19 [40]. Additionally, the early administration of the newer generation more potent $\mathrm{P} 2 \mathrm{Y}_{12}$ receptor inhibitors (e.g., prasugrel, ticagrelor) as well prolonged infusions of cangrelor or GP IIb/IIIa inhibitors may be considered after PCI to mitigate the high rates of thrombotic events observed. As we await data from robust clinical trials, it is reasonable to consider extended therapeutic anticoagulation in hospitalized patients with severe COVID-19, especially in those undergoing primary PCI for critically ill STEMI or NSTEMI/UA [41].

There is a growing body of evidence that ACS treatment is significantly impacted by the COVID-19 pandemic. De Luca et al. [42•] analyzed the large ISACS-STEMI COVID-19 registry in Europe which includes 77 centers in 18 different countries $(n=6609)$. When compared to pre-pandemic data, there was a significant reduction in primary PCI observed during the COVID-19 pandemic (incidence rate ratio: $0.811 ; 95 \% \mathrm{CI}$ : 0.78 to $0.84 ; p<0.0001)$. The investigators also reported increased total ischemic time and door-to-balloon times in STEMI patients during the pandemic timeframe after adjusting for baseline characteristics (adjusted OR: 1.34; 95\% CI: 1.13 to $1.58 ; p=0.001$ and $1.17 ; 95 \%$ CI: 1.05 to $1.29 ; p=0.003$, respectively). This highlights a potential gap 
in care which can be better addressed as HCWs get progressively vaccinated against COVID 19.

Depending on the hospital infrastructure, it may be reasonable to designate a specific catheterization laboratory specifically for COVID-19 patients to help limit HCWs exposure and expedite delivery of invasive care [43]. Evaluation of a patient's respiratory status, via physical examination and if possible, imaging (i.e., chest Xray or CT scan), should be performed prior to transferring the patient into the catheterization laboratory. If there is concern regarding the patient's respiratory status, intubation should be performed prior to the arrival of the patient to the catheterization laboratory [44]. Patients who are not intubated should be outfitted with a mask to limit exposure of airborne particles. All HCWs present for the case must have appropriate personal protective equipment (PPE), including facemasks (N95 respirators, or preferably powered air purifying respirators (PAPR)), face-shield or googles for eye protection, and contact isolation gowns $[43,45]$. The time limitation for appropriately donning PPE by all HCWs involved in the procedure should be taken into consideration when deciding to proceed with an invasive approach for ACS. Following completion of the procedure, patients should be transferred to postprocedure isolation rooms and a terminal cleaning of the catheterization laboratory should be performed. As such, it may be prudent to perform cases in COVID-19 suspected or positive cases towards the end of the working day, if feasible [43]. A summary of these recommendations can be found in Table 2 .

\section{Non-critically III STEMI}

In general, all STEMI patients can continue to follow a guideline-driven therapy of routine primary percutaneous

Table 2 Safety considerations for an invasive approach in COVID-19 suspected or positive patients ${ }^{16}$

Personal protective equipment

-Facemasks (N95 respirators, or PAPR)

- Goggles or eye-protection

-Gowns

Catheterization laboratory

-Intubation or additional respiratory support as needed prior to patient arrival in catheterization laboratory

-Perform any additional invasive procedures (e.g., intra-aortic balloon pump, mechanical support device, pulmonary artery catheter) in catheterization laboratory prior to transfer of patient

-Negative pressure room (if possible)

-Attempt to perform COVID-19 suspected or positive cases at the end of the working day (if possible)

-Terminal cleaning of catheterization laboratory room upon completion of case coronary intervention (PCI) without delay [33]. However, as the incidence of COVID-19 rises, a shift in practice methods should be considered in patients presenting with STEMI but are not critically ill (i.e., without cardiogenic shock, profound hemodynamic instability, refractory heart failure, mechanical complications, malignant ventricular arrhythmias, or cardiac arrest). In non-critically ill patients in whom there is a low suspicion of COVID-19 (either based on symptoms, history, or a negative COVID-19 test), a primary PCI approach should be used. Additionally, in patients whose ECG is concerning for a large area of myocardium in jeopardy (e.g., a large anterior or anteroseptal injury pattern), a primary PCI approach should be undertaken (Fig. 2).

On the other hand, in patients who present with possible STEMI and ambiguous clinical features (e.g., discordant clinical symptoms, diffuse ST segment elevations or nonclassic ECG findings), further noninvasive evaluation may be pursued. Additional ECGs, troponin levels, and an echocardiogram to detect regional wall motion abnormalities can aid the interventional care team in deciding whether or not to pursue an emergent invasive strategy. In these non-critically ill patients with STEMI, the overall clinical status of the patient must be carefully balanced against the potential exposure risk to $\mathrm{HCWs}$ and predicted clinical benefit to the patient $[43,46]$. In patients who are hemodynamically stable with suspected or positive test for COVID-19 and in patients in whom the risks of proceeding with an invasive approach outweigh its benefits or when primary PCI is not expected to be delivered in a timely manner ( $<120 \mathrm{~min}$ door-to-balloon time), it is appropriate to consider fibrinolytic therapy, in isolation or possibly as part of a pharmaco-invasive approach [32, 43, 47, 48]. A pharmaco-invasive approach would entail referral to the cardiac catheterization laboratory for PCI within 3-24 h after successful reperfusion (at which time testing for COVID-19 and appropriate precautionary measures to minimize HCW exposure are undertaken). A pharmacoinvasive strategy usually reduces recurrent ischemia and myocardial infarction in high-risk STEMI patients compared to a strategy of fibrinolytic therapy alone.

As the use of fibrinolytics have waned in recent years [49], it is important for hospitals to review and reenact protocols related to the administration of fibrinolytics and subsequent patients' monitoring and management [44]. Fibrinolytic therapy can be a viable alternative in patients who present early (within 2-3 h of symptom onset), are hemodynamically stable, and are suspected to have COVID-19. When choosing fibrinolytic therapy, it is preferred that the newer generation tenecteplase (TNK) is utilized, as it has been shown to have lower non-cerebral bleeding rates when compared to alteplase and is easier to administer (a weight-based bolus injection) [50]. Given the time sensitive nature of myocardial ischemia, 


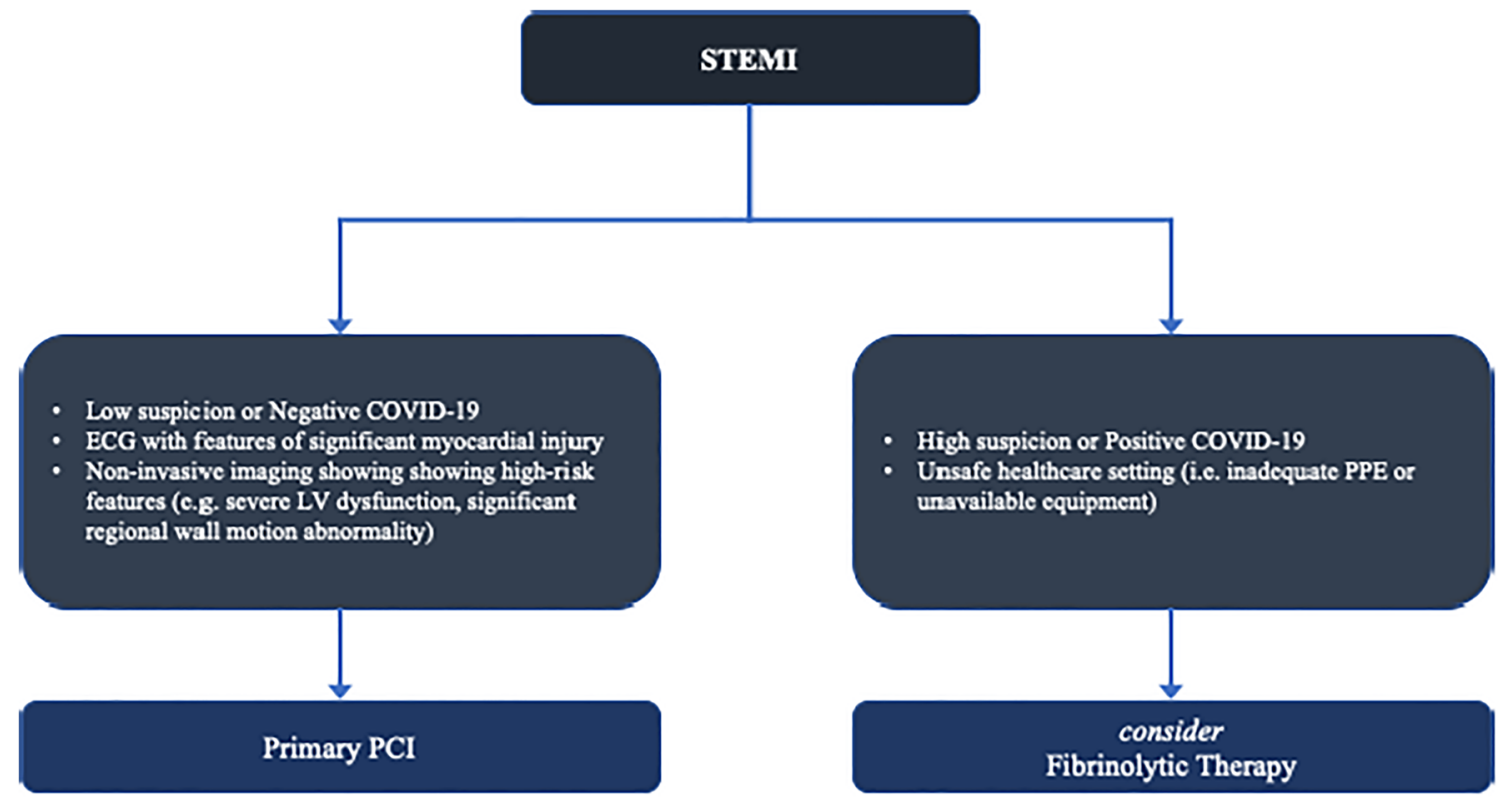

Fig. 2 Management algorithm for non-critically Ill STEMI patients

fibrinolytic therapy should be administered within a doorto-needle time (DNT) of $30 \mathrm{~min}$ or less [33]. Most importantly, the use of fibrinolytics has the potential to limit HCW exposure to COVID-19 and allow time to better evaluate the patient for COVID-19 with significantly reduced risks. Absolute and relative contradictions to fibrinolytics should be reviewed prior to their administrations (Table 3) [51]. Notably, there is a higher risk of intracranial hemorrhage in patients who receive fibrinolytic therapy versus PCI [52]. In certain patients with ACS mimickers (e.g., myocarditis, coronary spasm, cardiomyopathy) but

Table 3 Contraindications to fibrinolytic therapy in Patients with STEMI $^{22}$

\footnotetext{
Absolute contraindications

-Any prior intracranial hemorrhage

-Ischemic stroke within 3 months EXCEPT acute ischemic stroke within $3 \mathrm{~h}$

-Known structural cerebral vascular lesion (e.g., AVM)

-Closed head trauma or facial trauma within 3 weeks

-Known intracranial malignancy

-Suspected aortic dissection

-Active bleeding or known bleeding disorder

Relative contraindications

-History of chronic, severe, poorly controlled hypertension

-Severe uncontrolled hypertension on presentation (SBP $>180 \mathrm{mmHg}$ or DBP $>110 \mathrm{mmHg}$ )

- History of prior ischemic stroke $>3$ months

-Traumatic or prolonged (>10 min) CPR or major surgery within 3 weeks

-Recent (within 2-4 weeks) internal bleeding

-Non-compressible vascular punctures

-Pregnancy

-For streptokinase prior exposure ( $>5$ days ago) or prior allergic reaction
}

not true ACS, the administration of fibrinolytics results in increased bleeding risk without benefit. Fibrinolytic therapy should therefore be used judiciously.

\section{Non-critically III NSTEMI/UA}

In non-critically ill patients with NSTEMI/UA, a thorough initial assessment of the patient must be performed. In general, all patients should be presumed to be COVID-19 positive prior to any testing, given that a significant number of individuals with COVID-19 are asymptomatic [53]. Previous studies have shown that in high- and moderate-risk patients with non-STelevation ACS, an invasive strategy is superior to an ischemiaguided approach, and reduces the incidence of recurrent myocardial infarction [54]. In low-risk patients, an ischemia-guided approach is acceptable, and patients may cross over to an invasive strategy in case of significant spontaneous or inducible ischemia. Risk stratification can be performed using validated objective risk scores such as the Global Registry of Acute Coronary Events (GRACE) or TIMI (Thrombolysis in Myocardial Infarction) risks score. Further evaluation of patients with noninvasive testing such as echocardiography may help delineate high-risk (e.g., large myocardium in jeopardy, severe LV dysfunction, severe diastolic dysfunction) from low-risk patients $[43,47]$. In patients with high-risk features (e.g., dynamic ECG changes, rising troponin biomarkers) and those with elevated risk scores (e.g., GRACE score $>140$, or TIMI score $\geq 5$ ), an early invasive strategy within $12-24 \mathrm{~h}$ is recommended [34] (Fig. 3). In patients who develops signs of critical illness (e.g., refractory ischemic symptoms, hemodynamic or electrical instability), an immediate invasive strategy (within $\leq 2 \mathrm{~h}$ ) should be pursued. 


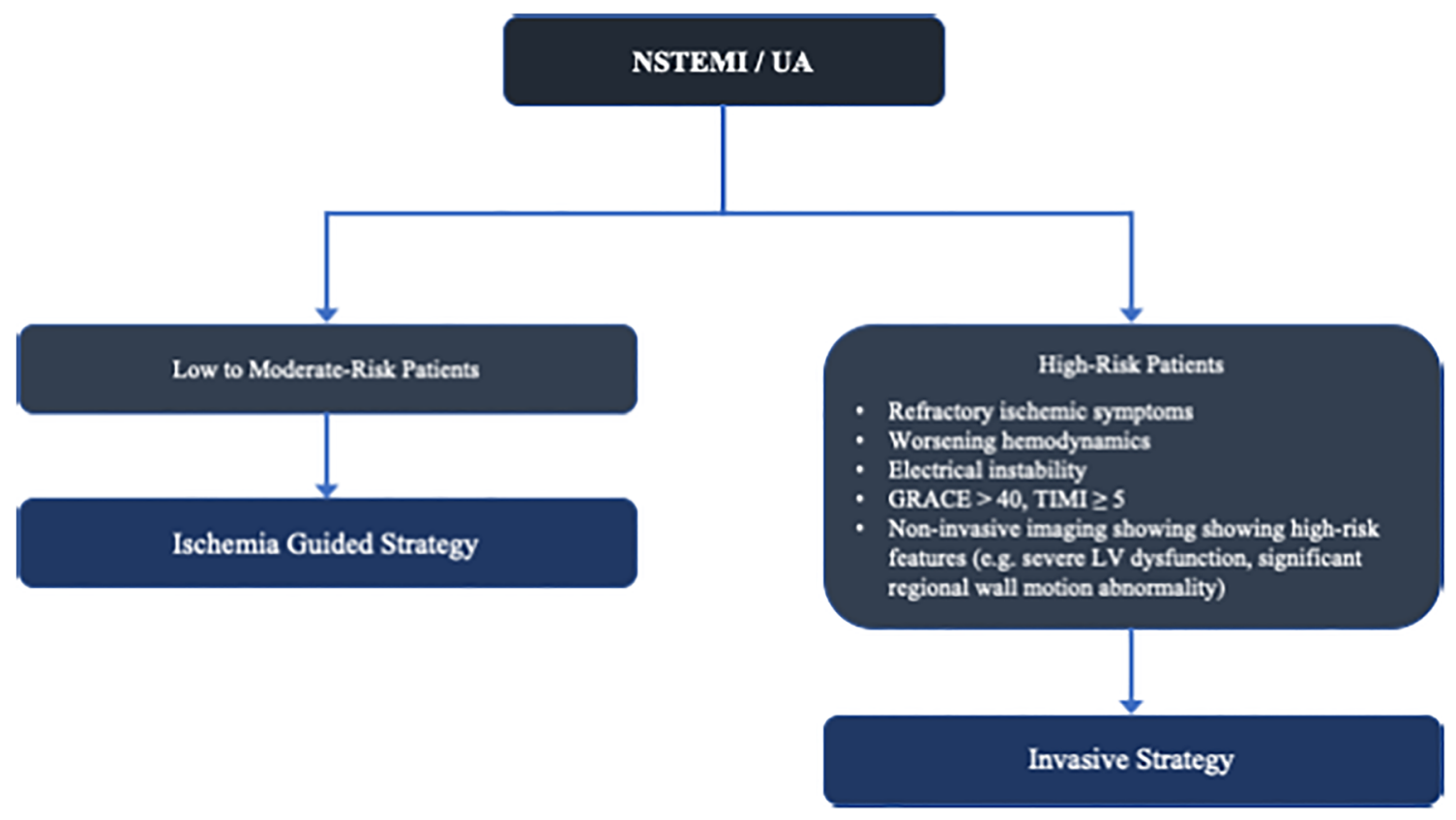

Fig. 3 Management algorithm for non-critically Ill NSTEMI/UA patients

In the COVID-19 era, it may be reasonable to employ an ischemia-guided strategy to patients determined to be at lowor moderate-risk, in whom aggressive implementation of guideline-directed medical therapies is advocated. Coronary angiography with intent to revascularize may be reserved, in the COVID-19 era, to patients at high-risk and to those who cannot be clinically stabilized [34]. Optimal medical therapy in non-PCI-treated patient may include the use of dual antiplatelet therapy - aspirin, a $\mathrm{P}_{2} \mathrm{Y}_{12}$ inhibitor (clopidogrel or ticagrelor) _ and a single anticoagulant (e.g., unfractionated heparin, enoxaparin, fondaparinux) [34].

\section{Conclusions}

As COVID-19 cases continue to rise and its burden on our health-care system takes its toll, cardiologists will have to make difficult decisions regarding the management of patients with ACS. In patients with STEMI or NSTEMI with very high-risk features (e.g., shock, ongoing ischemia, malignant arrhythmias), emergent PCI remains the treatment of choice. However, it is reasonable to consider fibrinolytic therapy in early presenters (within 2-3 h of symptom onset) with STEMI who are clinically stable. In low- and moderate-risk patients with NSTEMI or UA, an upfront ischemia-guided approach with guideline-directed medical therapies is reasonable, with the option for pursuing PCI based on patient stability and recurrence of ischemia. As the health-care community continues to gain more experience managing ACS in COVID19 patients and HCWs become vaccinated, we must evolve our practices to improve survival and quality of care for our patients while maintaining protocols to limit resource utilization and protect our HCWs.

\section{Declarations}

Human and Animal Rights and Informed Consent This article does not contain any studies with human or animal subjects performed by any of the authors.

Conflict of Interest The authors declare no competing interests.

\section{References}

Papers of particular interest, published recently, have been highlighted as:

- Of importance

- Of major importance

1. Dong E, Du H, Gardner L. An interactive web-based dashboard to track COVID-19 in real time. Lancet Infect Dis. 2020;20:533-4.

2. Modin D, Claggett B, Sindet-Pedersen C, Lassen MCH, Skaarup $\mathrm{KG}$, Jensen JUS, et al. Acute COVID-19 and the incidence of ischemic stroke and acute myocardial infarction. Circulation. 2020;142(21):2080-2.

3. Wu Z, McGoogan JM. Characteristics of and important lessons from the coronavirus disease 2019 (COVID-19) outbreak in China: summary of a report of 72314 cases from the Chinese Center for Disease Control and Prevention. JAMA. 2020;323: 1239-42. 
4. Badawi A, Ryoo SG. Prevalence of comorbidities in the Middle East respiratory syndrome coronavirus (MERS-CoV): a systematic review and meta-analysis. Int J Infect Dis. 2016;49:129-33.

5. Fisher DA, Lim T-K, Lim Y-T, Singh KS, Tambyah PA. Atypical presentations of SARS. Lancet. 2003;361:1740.

6. Yang J, Zheng Y, Gou X, Pu K, Chen Z, Guo Q, et al. Prevalence of comorbidities in the novel Wuhan coronavirus (COVID-19) infection: a systematic review and meta-analysis. Int J Infect Dis. 2020;94:91-5.

7. Gu T, Chu Q, Yu Z, Fa B, Li A, Xu L, et al. History of coronary heart disease increased the mortality rate of patients with COVID19: a nested case-control study. 2020;10(9):e038976. https://doi. org/10.1136/bmjopen-2020-038976

8. Guo T, Fan Y, Chen M, Wu X, Zhang L, He T, et al. Cardiovascular implications of fatal outcomes of patients with coronavirus disease 2019 (COVID-19). JAMA Cardiol. 2020;5(7):811-8.

9. Shi S, Qin M, Shen B, Cai Y, Liu T, Yang F, et al. Association of cardiac injury with mortality in hospitalized patients with COVID19 in Wuhan, China. JAMA Cardiol. 2020;5:802-10.

10. Nishiga M, Wang DW, Han Y, Lewis DB, Wu JC. COVID-19 and cardiovascular disease: from basic mechanisms to clinical perspectives. Nat Rev Cardiol. 2020;17(9):543-58.

11. Wang D, Hu B, Hu C, Zhu F, Liu X, Zhang J, et al. Clinical characteristics of 138 hospitalized patients with 2019 novel coronavirus-infected pneumonia in Wuhan, China. JAMA. 2020;323(11):1061-9.

12. Bhatla A, Mayer MM, Adusumalli S, Hyman MC, Oh E, Tierney A, et al. COVID-19 and cardiac arrhythmias. Heart Rhythm. 2020;17(9):1439-44.

13. Giustino G, Pinney SP, Lala A, Reddy VY, Johnston-Cox HA, Mechanick JI, et al. Coronavirus and cardiovascular disease, myocardial injury, and arrhythmia: JACC focus seminar. J Am Coll Cardiol. 2020;76(17):2011-23.

14. Lindner D, Fitzek A, Brauninger H, Aleshcheva G, Edler C, Meissner K, et al. Association of cardiac infection with SARSCoV-2 in confirmed COVID-19 autopsy cases. JAMA Cardiol. 2020;5(11):1281-5.

15. Inciardi RM, Lupi L, Zaccone G, Italia L, Raffo M, Tomasoni D, et al. Cardiac Involvement in a patient with coronavirus disease 2019 (COVID-19). JAMA Cardiol. 2020;5(7):819-24.

16. Kim IC, Kim JY, Kim HA, Han S. COVID-19-related myocarditis in a 21-year-old female patient. Eur Heart J. 2020;41(19):1859.

17. Escher F, Pietsch H, Aleshcheva G, Bock T, Baumeier C, Elsaesser A, et al. Detection of viral SARS-CoV-2 genomes and histopathological changes in endomyocardial biopsies. ESC Heart Fail. 2020;7(5):2440-7.

18. Garcia S, Albaghdadi MS, Meraj PM, Schmidt C, Garberich R, Jaffer FA, et al. Reduction in ST-segment elevation cardiac catheterization laboratory activations in the United States during COVID-19 pandemic. J Am Coll Cardiol. 2020;75(22):2871-2.

19. De Rosa S, Spaccarotella C, Basso C, Calabro MP, Curcio A, Filardi PP, et al. Reduction of hospitalizations for myocardial infarction in Italy in the COVID-19 era. Eur Heart J. 2020;41(22): 2083-8.

20. Baldi E, Sechi GM, Mare C, Canevari F, Brancaglione A, Primi R, et al. Out-of-hospital cardiac arrest during the Covid-19 outbreak in Italy. N Engl J Med. 2020;383(5):496-8.

21. Lai PH, Lancet EA, Weiden MD, Webber MP, Zeig-Owens R, Hall $\mathrm{CB}$, et al. Characteristics associated with out-of-hospital cardiac arrests and resuscitations during the novel coronavirus disease 2019 pandemic in New York City. JAMA Cardiol. 2020;5:1154.

22. Scquizzato T, Landoni G, Paoli A, Lembo R, Fominskiy E, Kuzovlev A, et al. Effects of COVID-19 pandemic on out-ofhospital cardiac arrests: a systematic review. Resuscitation. 2020;157:241-7.
23. Mogensen TH. Pathogen recognition and inflammatory signaling in innate immune defenses. Clin Microbiol Rev. 2009;22(2):240-73 Table of Contents.

24. Vallance P, Collier J, Bhagat K. Infection, inflammation, and infarction: does acute endothelial dysfunction provide a link? Lancet. 1997;349(9062):1391-2.

25. Thygesen K, Alpert JS, Jaffe AS, Chaitman BR, Bax JJ, Morrow DA, et al. Fourth Universal Definition of Myocardial Infarction (2018). J Am Coll Cardiol. 2018;72(18):2231-64.

26. Zheng Y-Y, Ma Y-T, Zhang J-Y, Xie X. COVID-19 and the cardiovascular system. Nat Rev Cardiol. 2020:1-2.

27. Mahmud E, Dauerman HL, Welt FGP, Messenger JC, Rao SV, Grines C, et al. Management of acute myocardial infarction during the COVID-19 pandemic: a consensus statement from the Society for Cardiovascular Angiography and Interventions (SCAI), the American College of Cardiology (ACC), and the American College of Emergency Physicians (ACEP). Catheter Cardiovasc Interv. 2020;96(2):336-45.

28. Temporary emergency guidance to STEMI systems of care during the COVID-19 pandemic: AHA's Mission: Lifeline. Circulation. 2020;142(3):199-202.

29. Cardiology TESf. ESC guidance for the diagnosis and management of $\mathrm{CV}$ disease during the COVID-19 pandemic [updated 10 June 2020. Available from: https://www.escardio.org/Education/ COVID-19-and-Cardiology/ESCCOVID-19-Guidance.

30. Gupta AK, Jneid H, Addison D, Ardehali H, Boehme AK, Borgaonkar $\mathrm{S}$, et al. Current perspectives on coronavirus disease 2019 and cardiovascular disease: a white paper by the JAHA Editors. J Am Heart Assoc. 2020;9(12):e017013.

31. Clerkin KJ, Fried JA, Raikhelkar J, Sayer G, Griffin JM, Masoumi A, et al. Coronavirus disease 2019 (COVID-19) and cardiovascular disease. Circulation. 2020;2019.

32. Driggin E, Madhavan MV, Bikdeli B, Chuich T, Laracy J, BondiZoccai G, et al. Cardiovascular considerations for patients, health care workers, and health systems during the coronavirus disease 2019 (COVID-19) pandemic. J Am Coll Cardiol 2020;2019.

33. Antman EM, Anbe DT, Armstrong PW, Bates ER, Green LA, Hand M, et al. ACC/AHA guidelines for the management of patients with ST-elevation myocardial infarction: a report of the American College of Cardiology/American Heart Association Task Force on Practice Guidelines (Committee to Revise the 1999 Guidelines for the Managem). J Am Coll Cardiol. 2004;44: E1-E211.

34. Amsterdam EA, Wenger NK, Brindis RG, Casey DE Jr, Ganiats TG, Holmes DR Jr, et al. 2014 AHA/ACC guideline for the management of patients with non-ST-elevation acute coronary syndromes: a report of the American College of Cardiology/ American Heart Association Task Force on Practice Guidelines. J Am Coll Cardiol. 2014;64(24):e139-228.

35. Stefanini GG, Montorfano M, Trabattoni D, Andreini D, Ferrante G, Ancona M, et al. ST-elevation myocardial infarction in patients with COVID-19: clinical and angiographic outcomes. Circulation. 2020;141(25):2113-6.

36. Bangalore S, Sharma A, Slotwiner A, Yatskar L, Harari R, Shah B, et al. ST-Segment Elevation in Patients with Covid-19 - A Case Series. N Engl J Med. 2020;382(25):2478-80. https://doi.org/10. 1056/NEJMc2009020

37.• Hamadeh A, Aldujeli A, Briedis K, Tecson KM, Sanz-Sanchez J, Al Dujeili M, et al. Characteristics and outcomes in patients presenting with COVID-19 and ST-segment elevation myocardial infarction. Am J Cardiol. 2020;131:1-6. This study demonstrated that the majority of patients with COVID-19 and STEMI were treated with fibrinolytic therapy. Additionally, in patients who underwent PCI, the rates of in-sent thrombosis was high. 
38. Klok FA, Kruip M, van der Meer NJM, Arbous MS, Gommers D, Kant KM, et al. Incidence of thrombotic complications in critically ill ICU patients with COVID-19. Thromb Res. 2020;191:145-7.

39. Sudhakar D, Jneid H, Lakkis N, Kayani WT. Primary percutaneous coronary intervention or fibrinolytic therapy in COVID 19 patients presenting with ST-segment elevation myocardial infarction. Am J Cardiol. 2020;134:158.

40. Paranjpe I, Fuster V, Lala A, Russak AJ, Glicksberg BS, Levin MA, et al. Association of treatment dose anticoagulation with in-hospital survival among hospitalized patients with COVID-19. J Am Coll Cardiol. 2020;76(1):122-4.

41. Chowdhury JF, Moores LK, Connors JM. Anticoagulation in hospitalized patients with Covid-19. N Engl J Med. 2020;383(17): 1675-8.

42. De Luca G, Verdoia M, Cercek M, Jensen LO, Vavlukis M, Calmac L, et al. Impact of COVID-19 pandemic on mechanical reperfusion for patients with STEMI. J Am Coll Cardiol. 2020;76(20):2321-30. The investigators in this study not only found a decreased rate of STEMI in the COVID-19 pandemic, the findings also demonstrate an increased total ischemic time and door-to-balloon times in STEMI patients during the pandemic timeframe after adjusting for baseline characteristics.

43. Welt FGP, Shah PB, Aronow HD, Bortnick AE, Henry TD, Sherwood MW, et al. Catheterization laboratory considerations during the coronavirus (COVID-19) pandemic: from ACC's Interventional Council and SCAI. J Am Coll Cardiol. 2020;75: 2372-5.

44. Szerlip M, Anwaruddin S, Aronow HD, Cohen MG, Daniels MJ, Dehghani $\mathrm{P}$, et al. Considerations for cardiac catheterization laboratory procedures during the COVID-19 pandemic perspectives from the Society for Cardiovascular Angiography and Interventions Emerging Leader Mentorship (SCAI ELM) members and graduates. Catheter Cardiovasc Interv. 2020;96:586-97.

45. (WHO) WHO, editor Rational use of personal protective equipment for coronavirus disease 2019 ( COVID-19 ). Who; 2020: World Health Organization.
46. Harrison D, Muradali K, El Sahly H, Bozkurt B, Jneid H. Impact of the SARS-CoV-2 pandemic on health-care workers. Hosp Pract (1995). 2020;48(4):161-4.

47. Zeng J, Huang J, Pan L. How to balance acute myocardial infarction and COVID-19: the protocols from Sichuan Provincial People's Hospital. Intensive Care Med 2020:10-2.

48. Jing Z-C, Zhu H-D, Yan X-W, Chai W-Z, Zhang S. Recommendations from the Peking Union Medical College Hospital for the management of acute myocardial infarction during the COVID-19 outbreak. Eur Heart J. 2020;41:1791-4. https://doi. org/10.1093/eurheartj/ehaa258

49. Elbadawi A, Mahtta D, Elgendy IY, Saad M, Krittanawong C, Hira RS, et al. Trends and outcomes of fibrinolytic therapy for STEMI: insights and reflections in the COVID-19 Era. JACC Cardiovasc Interv. 2020;13(19):2312-4.

50. Van de Werf F. Single-bolus tenecteplase compared with frontloaded alteplase in acute myocardial infarction: the ASSENT-2 double-blind randomised trial. Lancet. 1999;354:716-22.

51. O'Connor RE, Brady W, Brooks SC, Diercks D, Egan J, Ghaemmaghami C, et al. Part 10: acute coronary syndromes: 2010 American Heart Association guidelines for cardiopulmonary resuscitation and emergency cardiovascular care. Circulation. 2010;122:S787-817.

52. Armstrong PW, Gershlick A, Goldstein P, Wilcox R, Danays T, Bluhmki E, et al. The strategic reperfusion early after myocardial infarction (STREAM) study. Am Heart J. 2010;160:30-5.

53. Nikolai LA, Meyer CG, Kremsner PG, Velavan TP. Asymptomatic SARS Coronavirus 2 infection: Invisible yet invincible. Int J Infect Dis. 2020;100:112-6.

54. Fox KAA, Clayton TC, Damman P, Pocock SJ, de Winter RJ, Tijssen JGP, et al. Long-term outcome of a routine versus selective invasive strategy in patients with non-ST-segment elevation acute coronary syndrome: a meta-analysis of individual patient data. J Am Coll Cardiol. 2010;55:2435-45.

Publisher's Note Springer Nature remains neutral with regard to jurisdictional claims in published maps and institutional affiliations. 